\title{
lieuxdits \#6
}

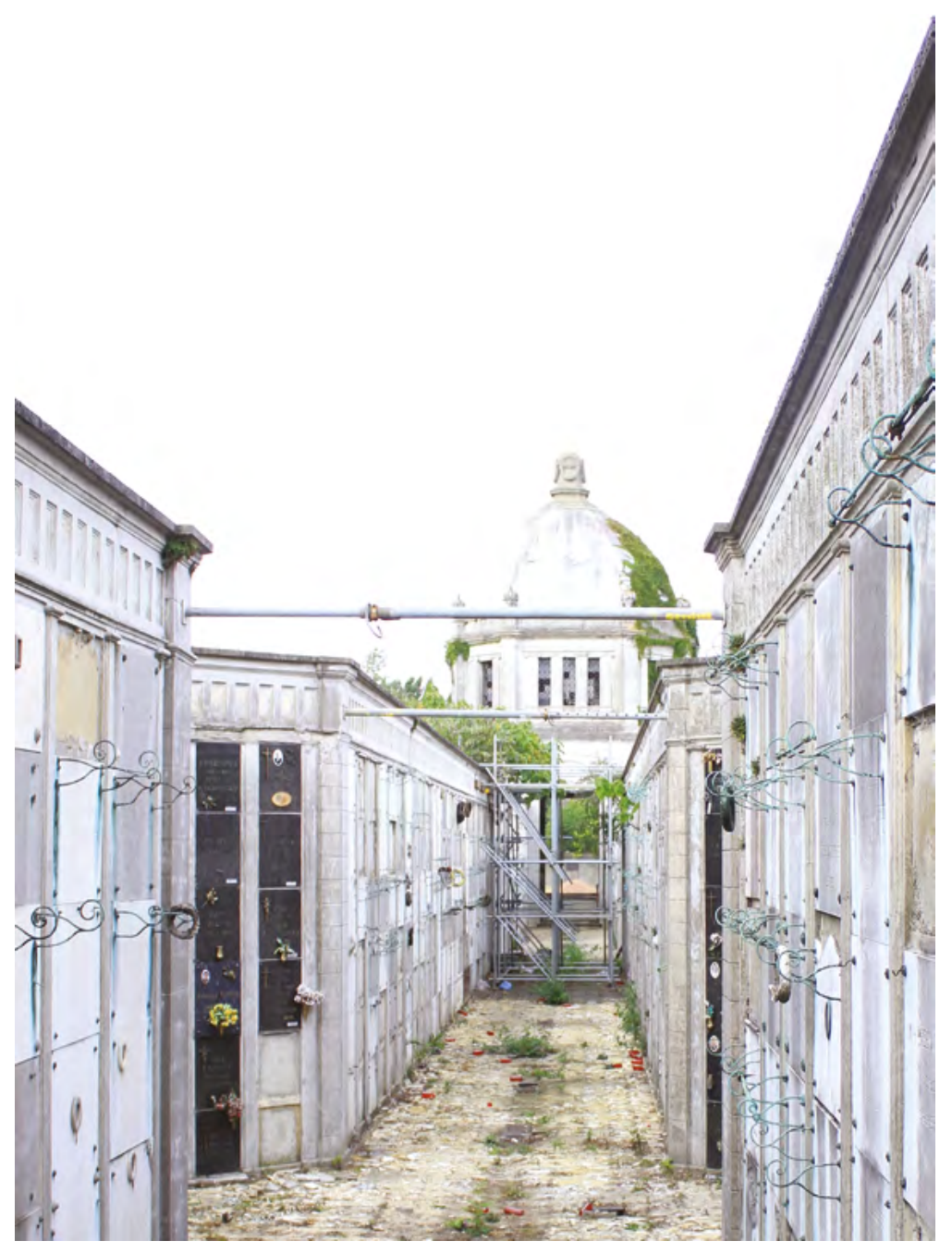

Référence bibliographique :

Christine Fontaine et Catherine Vanhamme, "Édito", lieuxdits\#6, novembre 2013, p.2.

\section{La revue lieuxdits}

Faculté d'architecture, d'ingénierie architecturale, d'urbanisme (LOCI)

Univesrité catholique de Louvain (UCL).

Éditeur responsable : Jean-Paul Verleyen, place des Sciences, 1 - 1348 Louvain-la-Neuve

Comité de rédaction : Martin Buysse, Damien Claeys, Gauthier Coton,

Jean-Philippe De Visscher, Guillaume Vanneste, Jean-Paul Verleyen

Conception graphique : Nicolas Lorent

Impression : école d'imprimerie Saint-Luc Tournai

\section{(c) (1) () $\Theta$}




\section{SCENARIO POUR UNE REFORME EN MOUVEMENT}

Au lendemain des élections des représentants aux commissions de programmes BARC-MARC, écrire cet édito est forcément un acte politique. Les politiques ont eu tendance à promettre le 'changement' comme solution à tout problème. Or ici, le changement est l'objet dont il faut trouver la mesure.

La notion de changement est inhérente au vivant. Il en va de même pour les sociétés et bien entendu pour l'enseignement : adaptations aux réformes pédagogiques, au cadre politique et économique, mais aussi aux ambitions de ses acteurs.

L' ex-doyen de la faculté de planification à l'IUAV, Domenico Patassini, nous renvoyait à l'idée de scénario qu'il appliqua dans le cadre des réformes de son institution. Il partait de trois axes :

- l'un fondé sur les prévisions ou tendances ;

- l'autre comme projection d'objectifs maximum et minimum acceptables sous la contrainte;

- le dernier comme volonté ou désir, idéal et indépendant.

Définir notre scénario dans l'espace des possibles de ces trois axes, permettrait de pointer un plan de route. Parfois, nous préférons un rêve à une réalité brute, parfois nous oublions le rêve en raison de contraintes, enfin nous essayons de tirer les leçons du passé ou de garder sous contrôle les risques à venir.

Axe 1 : les prévisions ou le changement dans la continuité.

Voici trois ans, les commissions de programmes s'attelaient, simultanément à la création de la faculté et à la redéfinition des programmes d'enseignement de l'architecture. Années durant lesquelles il a fallu se faire violence pour questionner nos pratiques pédagogiques et tenter de construire une nouvelle structure. La réforme est en route, engagée dans un programme commun à la rentrée 2012 sur les sites de Bruxelles et Tournai, dispensant tous deux le diplôme d'architecte. Nous tenons à remercier vivement Anne Croegaert, François Nizet, Jean-Louis Vanden Eynde et Bernard Wittevrongel qui ont mené ce projet jusqu'à un cadre structuré du programme bachelier. À nous de viser un programme pour les masters.

Axe 2 : la projection ou la liberté trouvée entre les lignes de contraintes.

Réformer le programme master, c'est assurer le cadre qui amène les étudiants à la pratique de l'architecture, mais c'est aussi favoriser des liens avec la recherche.

L'occasion est de révéler les spécificités de chaque site, de favoriser les mobilités intra-, intersites et internationales, tant au niveau des enseignants que des étudiants. Les prochaines années seront également le temps de l'évaluation et de la consolidation de ces programmes, mélanges de traditions, d'expériences mais aussi, heureusement, de certaines inventions pédagogiques.

Axe 3 : le rêve pour porter les acteurs de la réforme.

Le rêve de devenir passe nécessairement par la question des possibles et l'écoute des visions. Une part sera laissée à l'expérience, au génie, à l'intuition. La connaissance mutuelle sera l'esprit par lequel nous passerons pour construire un ensemble de relations et de programmes porteurs de sens. Nous continuerons ainsi à modeler l'identité de LOCI avec nos collègues ingénieurs architectes et urbanistes.

Tout changement implique un état 'avant' et un état 'après'. Dans le cas de LOCI, préexistaient trois états et un avenir commun. Nous devons prendre conscience de la portée de ce qu'implique ce passage. Chacun des trois sites évoluait avec le temps, parfois par à-coups de bouleversements provoqués par décrets et réformes. Comme ces évolutions passées, l'intégration à l'université est une chance à saisir pour nous ajuster, entre nous, mais aussi au contact des enjeux contemporains au cœur desquels nous projetons notre avenir. 